\title{
Fluoroquinolone Resistance Among Gram-Negative Urinary Tract Patho- gens: Global Smart Program Results, 2009-2010
}

\author{
Sam Bouchillon ${ }^{{ }^{*}}$, Daryl J. Hoban ${ }^{1}$, Robert Badal ${ }^{1}$, and Stephen Hawser ${ }^{2}$ \\ ${ }^{1}$ International Health Management Associates, Inc., Schaumburg, IL, 60178 USA \\ ${ }^{2}$ IHMA Europe Sàrl, Rote de la Corniche 4, 1066 Epalinges, Switzerland
}

\begin{abstract}
OBJECTIVES: To determine the rates of fluoroquinolone resistant (FQR) in gram-negative bacilli urinary tract infections (UTIs) in a global population. METHODS: The Study for Monitoring Antimicrobial Resistance Trends (SMART) collected 1,116 FQR gram-negative urinary pathogens from hospitalized patients in 33 countries during 20092010. Amikacin, ertapenem, and imipenem were the most active agents tested against FQR UTI pathogens, including extended-spectrum beta-lactamase producers. RESULTS: FQR rates vary widely country to country with a range of $6 \%$ to 75\%. Regional FQR rates were 23.5\% in North America, 29.4\% in Europe, 33.2\% in Asia, 38.7\% in Latin America, and 25.5\% in the South Pacific. CONCLUSIONS: These observations suggest that fluoroquinolones may no longer be effective as first-line therapy for gram-negative UTI in hospitalized patients.
\end{abstract}

Keywords: Ertapenem, Fluoroquinolone resistance, Imipenem, SMART Global Surveillance, Urinary Tract Infection.

\section{INTRODUCTION}

Fluoroquinolone resistance in UTI pathogens has been increasing globally $[1,2]$. Poor health, urinary catheterization, recent hospitalization, and previous UTI are risk factors associated with increased fluoroquinolone resistance [3-5]. Independent risk factors also include prior exposure to antimicrobial agents including trimethoprim-sulfamethoxazole, metronidazole, cephalosporins, and fluoroquinolones [3-5]. Additionally, the increasing prevalence of beta-lactamases, including the global spread of CTX-M beta-lactamases with frequent cross-resistance with fluoroquinolones has become a major concern [6-9]. The Study for Monitoring Antimicrobial Resistance Trends (SMART) has tracked resistance in gram-negative aerobic pathogens of intra-abdominal infections since 2002, and in 2009 began including isolates from urinary tract infections (UTI). Evidence for increasing fluoroquinolone resistance in UTI is presented.

\section{MATERIAL AND METHODS}

A total of 98 investigational sites (Africa 1, Asia 19, Europe 27, Middle East 1, North America 29, Latin America 14, and South Pacific 7) from 33 countries each contributed up to 50 gram-negative UTI isolates from hospitalized patients in 2009 and 2010. The isolates collected were nonduplicate, consecutive isolates from hospitalized patients with UTI. All isolates were gram-negative isolates deemed to be clinically significant $\left(>10^{5} \mathrm{cfu} / \mathrm{ml}\right)$ by the participating site. Only one isolate per patient was accepted. The only

\footnotetext{
*Address correspondence to this author at the International Health Management Associates, Inc. 2122 Palmer Drive, Schaumburg, IL 60173 USA; Tel: +1 615.599.8429; Fax: +1 847.303.5601;

E-mail: sbouchillon@ihmainc.com
}

patient-specific data collected was age and gender. Isolates were further classified as hospital-associated (HA) or community-associated (CA) if the specimen was obtained $\geq 48$ hours or $<48$ hours after admission, respectively. Isolates were identified to the species level at each participating site and submitted to the central reference study center (Laboratories International for Microbiology Studies, a subsidiary of International Health Management Associates, Inc., Schaumburg, IL, USA) for identification confirmation and susceptibility testing. In 2009-2010, 3,845 gram-negative bacilli were submitted of which 1,116 were fluoroquinoloneresistant (FQR; resistant to both levofloxacin and ciprofloxacin).

Minimum inhibitory concentrations (MICs) were determined at the central laboratory using MicroScan ${ }^{\circledR}$ dehydrated microdilution panels (Siemens Medical Solutions Diagnostics, West Sacramento, CA, USA). Drug susceptibilities were defined using the Clinical and Laboratory Standards Institute M100-S20-U interpretive criteria breakpoints [10]. Quality control was performed on each day of testing using the CLSI recommended QC strains: Escherichia coli ATCC 25922, E. coli ATCC 35218, Pseudomonas aeruginosa ATCC 27853 and Klebsiella pneumoniae ATCC 700603 (positive ESBL control). Isolates were classified as ESBL producers when there was at least an eight fold reduction of the MICs for ceftazidime and/or cefotaxime tested in combination with clavulanic acid compared with the MICs when tested alone [10]. Significance was determined by Fisher's Exact Test, two-tailed.

\section{RESULTS}

The 3,845 urinary tract infection (UTI) isolates included 33 species of which the top 12 most frequently encountered 
species $(\mathrm{n}>20)$ comprised $>98 \%(3,781)$ of all isolates collected. The majority of the isolates (59.6\%) were collected from North America and Europe. The remaining isolates originated from Asia (17.6\%), Latin America (13.8\%), South Pacific (7.5\%), the Middle East (0.8\%), and Africa (0.6\%). Overall, females represented $65 \%$ of the patients. Almost half the isolates were from elderly patients $>65$ years of age $(1,845,49 \%)$ and $<10 \%$ (359) were from pediatric patients (0-16 years) with a mean age of 58.1 years for all patients represented. Isolates were evenly distributed between HA and CA UTIs, $38.4 \%$ versus $38.7 \%$, respectively ( $p>0.05$, Table 1). The 1,116 FQR isolates were evenly distributed between HA and CA populations as were the 381 fluoroquinolone-resistant extended-spectrum beta-lactamase producing $(\mathrm{ESBL}+)$ isolates $(\mathrm{p}>0.05)$.

Eleven of the top twelve UTI pathogens demonstrated resistance to the fluoroquinolones in varying degrees; only Citrobacter koseri remained 100\% (45/45; 95\% confidence interval $=91 \%-100 \%$ ) susceptible to fluoroquinolones. The in vitro percents susceptible for all study drugs against $\mathrm{FQR}$ isolates are presented in Table 2 . Overall, only amikacin, the carbapenems, and piperacillin-tazobactam inhibited $>75 \%$ of all $\mathrm{FQR}$ isolates.

Only ertapenem and imipenem inhibited $>95 \%$ of $\mathrm{FQR}$ E. coli including both ESBL+ and ESBL- strains. The least active agent was ampicillin-sulbactam. Amikacin susceptibility percentages were $>90 \%$ for $C$. freundii $(100 \%)$, Enterobacter aerogenes (100\%), E. coli, ESBL- (97\%), Klebsiella oxytoca, ESBL- (100\%), Morganella morganii
$(100 \%)$, and Proteus mirabilis, (93\%). Ertapenem and imipenem were particularly active against FQR ESBL+ and ESBL- E. coli with susceptibility percentages ranging from $95 \%$ to $100 \%$. None of the study drugs inhibited $>79 \%$ (imipenem) of FQR K. pneumoniae. Only ertapenem demonstrated consistent activity against $P$. mirabilis, inhibiting $100 \%$ of all isolates including both ESBL+ and ESBLstrains.

FQR rates varied widely among regions and countries. Regionally, FQR rates were: Latin America (38.7\%); Asia (33.2\%); Europe (29.4\%); South Pacific (25.5\%); Africa (25\%; one country only); North America (23.5\%); and Middle East $(20.7 \%)$. There was much wider variance among individual countries. $\mathrm{FQR}$ rates ranged from a low of $6 \%$ in Estonia and the United Kingdom (1 site each) to $75 \%$ in India (3 sites) (Fig. 1). The United States, contributing the most isolates from the largest number of sites $(1,026$ isolates, 23 sites), had a FQR rate for all UTI isolates of $24 \%$.

\section{DISCUSSION}

The SMART program has analyzed trends in antibiotic resistance in gram-negative bacilli isolated from patients with intra-abdominal infections since 2002 and, beginning in 2009, global trends in resistance in gram-negative bacilli in hospitalized patients with urinary tract infections. This study confirms the prevalence of UTIs in females compared to males in a 2:1 ratio previously reported [11] and may reflect the observation that females are more prone to contract UTIs as compared to males.

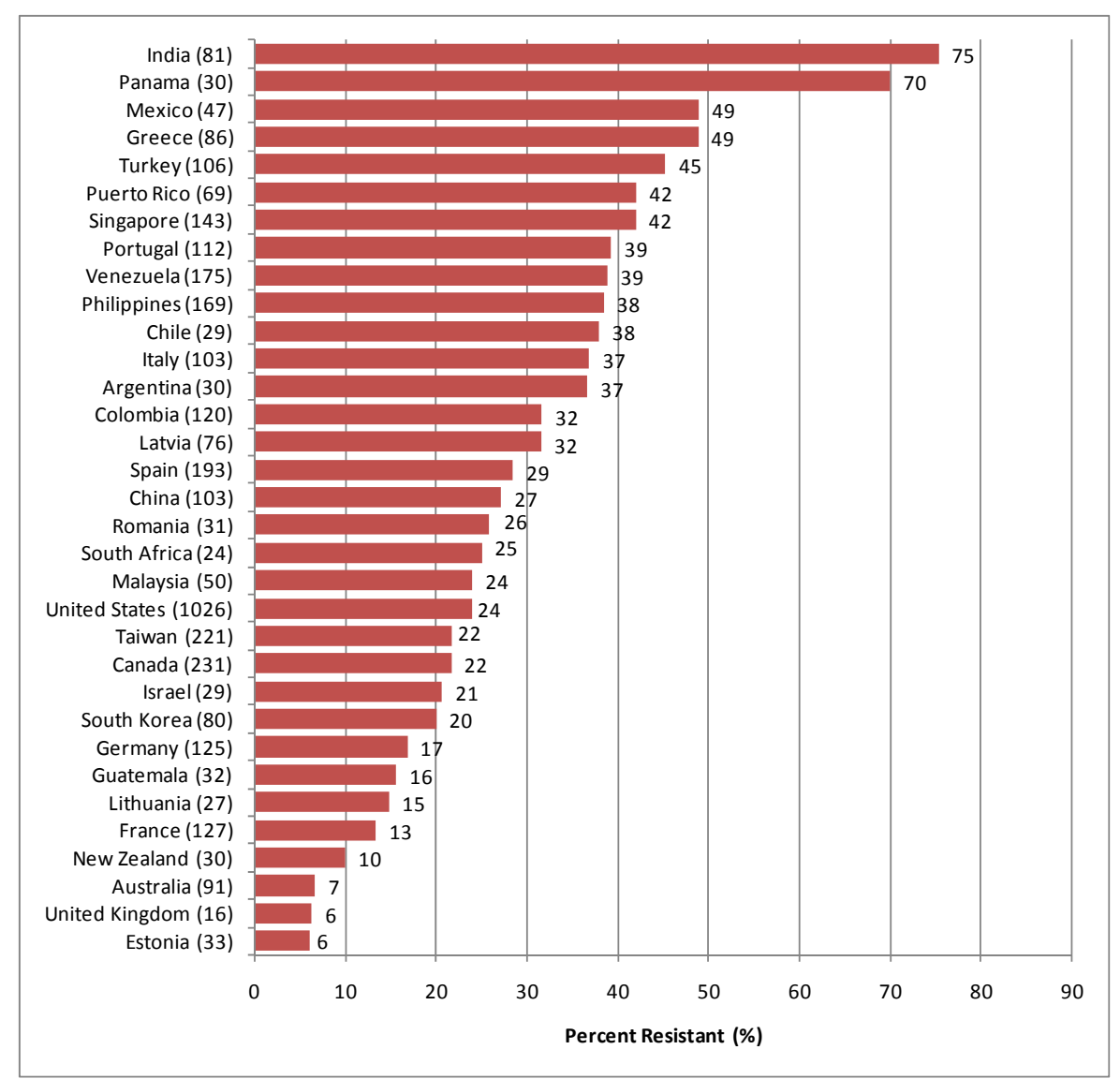

Fig. (1). Percent fluoroquinolone resistance* in 3,845 UTIs by country (n). 
A distinction was made in the collection time of the UTI specimen to categorize the infection as HA (specimen collection $\geq 48$ after admission) or CA (specimen collection $<48$ hrs after admission). Although there was a statistically significant difference in the percentage of ESBL+ isolates between HA (47.8\%) and CA (33.8\%) UTIs $(\mathrm{p}<0.001)$, there were no significant differences in the percentages of fluoroquinolone-resistant ESBL+ UTIs for HA (44.6\%) and CA $(36.8 \%)(p>0.05)$. This may reflect the growing numbers of community-acquired ESBLs containing the CTX-M-15 ESBL genotype that is strongly associated with multi-drug resistant phenotypes including fluoroquinolone resistance $[12,13]$.

The in vitro activity of the drugs in this study suggests that there are relatively few therapy alternatives for treatment of fluoroquinolones-resistant gram-negative UTI pathogens. Low susceptibility rates were seen for ampicillin-sulbactam, cefotaxime, cefoxitin, ceftazidime, and ceftriaxone against the majority of isolates. Only ertapenem and imipenem demonstrated consistent activity against ESBL+ isolates, with both equally active against ESBL $+E$. coli, imipenem more active against ESBL $+K$. oxytoca, and ertapenem more active against ESBL $+P$. mirabilis. None of the study drugs were more than $88 \%$ active (imipenem) against all $K$. pneumoniae. Overall, amikacin and piperacillin-tazobactam had similar in vitro activity to ertapenem and imipenem against all $\mathrm{FQR}$ isolates combined.

Fluoroquinolone resistance varied from country to country and less so, but significantly nevertheless, from region to region. The highest regional $\mathrm{FQR}$ rate was seen in Latin America at $38.7 \%$, but resistance was as high as $70 \%$ in one hospital in Panama and above 40\% from three sites in Puerto Rico and Mexico. The highest fluoroquinolone resistance rates in this study were seen in India where $75 \%$ of all UTIs were non-susceptible to the fluoroquinolones. The average for the Asian countries was $33.2 \%$. Fluoroquinolone resistance rates for Canada and the United States were 22\% and $24 \%$, respectively, and were more than double the rate reported as recently as 2006 by Karlowsky et al., in 1,858 E. coli [14]. Notably, the current rate of $49 \%$ resistance seen in Turkey is also almost double the $25 \%$ rate reported for that region during the same 2005-2006 time frame but that report was limited to E. coli only [1]. The lowest rates reported in this study were seen in Estonia and the United Kingdom $(6 \%)$, however, the significance of this is diminished due to the low n's and the fact that the isolates were collected from a single lab in each country.

This study is limited by four factors: (1) inconsistency in the sites reporting from 2009 and 2010 with only about half of the sites participating in both years; (2) the number of sites per county is limited, averaging 3 per country, and 13 countries having a single investigative site; (3) the lack of molecular characterization of resistance mechanisms limits the depth, but not necessarily the overall breadth of a surveillance of this type; and (4) the exclusion of useful UTI antimicrobials such as colistin, the tetracyclines, and trimethoprim-sulfamethoxazole in the evaluation. On the other hand, since all patients in this study were hospitalized patients, it is likely the majority of the UTIs were complicated, not uncomplicated, and the use of oral agents in such cases would have limited utility.

Fluoroquinolone resistance is increasing in UTI gramnegative pathogens both locally and regionally $[1,2,5,11]$. The SMART program plays a significant role in monitoring the evolution of resistance and emergence of specific resistance mechanisms and phenotypes. Close monitoring of resistance patterns may prove useful in directed empiric therapy not just in the treatment of UTI but other infections as well. Although in vitro data do not always equate to clinical outcomes, especially in UTIs where the fluoroquinolones often achieve high concentrations, this study suggests that alternatives to fluoroquinolone therapy may deserve consideration in environments of increasing fluoroquinolone resistance.

\section{FUNDING}

The SMART surveillance program is funded by Merck \& Co., Inc.

\section{ACKNOWLEDGEMENTS}

We thank all the SMART investigators for their participation in this program.

Table 1. Distribution of Isolates between Hospital and Community Associated Urinary Tract Infections Traparancy delavation

\begin{tabular}{|c|c|c|c|c|c|c|c|c|}
\hline \multirow{2}{*}{ Specimen Collection* } & \multicolumn{2}{|c|}{ All UTI Isolates } & $\begin{array}{c}\text { Fluoroquinolone Resistant UTI } \\
\text { Isolates }\end{array}$ & \multicolumn{3}{c|}{$\begin{array}{c}\text { All UTI ESBL+ } \\
\text { Fluoroquinolone Resis- } \\
\text { tant UTI ESBL+ }\end{array}$} \\
\cline { 2 - 9 } & $\mathbf{N}$ & \% of Total & $\mathbf{N}$ & \% of Total & N & \% of Total & N & \% of Total \\
\hline \hline$\geq 48$ hrs (HA) & 1476 & $38.4 \%$ & 454 & $40.7 \%$ & 255 & $47.8 \%$ & 170 & $44.6 \%$ \\
\hline$<48$ hrs (CA) & 1488 & $38.7 \%$ & 413 & $37.0 \%$ & 180 & $33.8 \%$ & 140 & $36.8 \%$ \\
\hline None Given & 881 & $22.9 \%$ & 249 & $22.3 \%$ & 98 & $18.4 \%$ & 71 & $18.6 \%$ \\
\hline Total & 3845 & & 1116 & & 533 & & 381 & \\
\hline p-Value $\dagger$ & $>0.05$ & & $>0.05$ & & $<0.001$ & & $>0.05$ & \\
\hline
\end{tabular}

* HA, hospital-associated; CA, community-associated.

$\dagger>0.05$, not statistically significant; $<0.001$, statistically significant (Fisher's Exact Test, two-tailed). 
Table 2. Percents Susceptible (\%) of FQR Urinary Tract Pathogens $(n=1,100)$

\begin{tabular}{|c|c|c|c|c|c|c|c|c|c|c|}
\hline $\begin{array}{c}\text { Organism* (FQR N/Total } \\
\text { N Tested) }\end{array}$ & AK & A/S & CPE & CTF & CFX & CAZ & CAX & ETP & IMP & $\mathbf{P} / \mathbf{T}$ \\
\hline A. baumannii $(30 / 57)$ & 37 & 23 & 7 & 3 & na & 7 & 7 & na & 43 & 10 \\
\hline C. freundii $(5 / 50)$ & 100 & 20 & 80 & 40 & 0 & 40 & 40 & 80 & 80 & 60 \\
\hline E. aerogenes $(2 / 58)$ & 100 & 0 & 50 & 0 & 50 & 0 & 0 & 50 & 100 & 50 \\
\hline E. coli $(763 / 2163)$ & 93 & 14 & 65 & 55 & 76 & 56 & 56 & 96 & 99 & 86 \\
\hline $\mathrm{ESBL}+(300 / 367)$ & 88 & 7 & 12 & 0 & 74 & 0 & 0 & 95 & 100 & 81 \\
\hline ESBL- (463/1796) & 97 & 19 & 99 & 91 & 77 & 92 & 92 & 97 & 99 & 89 \\
\hline K. oxytoca $(8 / 86)$ & 87 & 0 & 38 & 25 & 87 & 25 & 25 & 87 & 100 & 38 \\
\hline K. pneumoniae $(96 / 582)$ & 74 & 4 & 26 & 18 & 48 & 21 & 17 & 57 & 79 & 34 \\
\hline $\mathrm{ESBL}+(65 / 135)$ & 78 & 0 & 8 & 0 & 51 & 0 & 0 & 55 & 88 & 25 \\
\hline ESBL- $(31 / 447)$ & 65 & 13 & 65 & 55 & 42 & 65 & 52 & 61 & 61 & 52 \\
\hline M. morganii (8/49) & 100 & 0 & 38 & 38 & 50 & 50 & 38 & 100 & 50 & 100 \\
\hline P. mirabilis $(38 / 209)$ & 84 & 37 & 81 & 55 & 73 & 60 & 55 & 100 & 27 & 90 \\
\hline $\mathrm{ESBL}+(10 / 18)$ & 60 & 30 & 40 & 0 & 80 & 0 & 0 & 100 & 20 & 100 \\
\hline ESBL- $(28 / 191)$ & 93 & 39 & 96 & 75 & 71 & 82 & 75 & 100 & 29 & 86 \\
\hline P. aeruginosa $(124 / 295)$ & 62 & na & 31 & 7 & na & 49 & 8 & na & 54 & 73 \\
\hline
\end{tabular}

* C. koseri $(\mathrm{n}=45)$ not included as all were fluoroquinolone-susceptible.

AK, Amikacin; A/S, Ampicillin-Sulbactam; CPE, Cefepime; CTF, Cefotaxime; CFX, Cefoxitin; CAZ, Ceftazidime; CAX, Ceftriaxone; ETP, Ertapenem; IMP, Imipenem; P/T, Piperacillin-Tazobactam. na, CLSI breakpoints not available for this species/drug combination (na's were calculated as resistant for purposes of Grand Total percentages).

\section{CONFLICT OF INTEREST}

S. Bouchillon, D. Hoban, S. Hawser, and R. Badal served as scientific advisors or consultants to Merck, and received research support from Merck to conduct this study.

\section{TRANSPARENCY DECLARATION}

The authors are responsible for the work described in this paper. All authors were involved in at least one of the following: conception, design, acquisition, general analysis, statistical analysis, interpretation of data, and drafting the manuscript and/or revising the manuscript for important intellectual content. All authors provided final approval of the version to be published.

\section{REFERENCES}

[1] Aypak C, Altunsoy A, Duzgun N. Empiric antibiotic therapy in acute uncomplicated urinary tract infections and fluoroquinolone resistance: a prospective observational study. Ann Clin Microbiol Antimicrob $2009 ; 8: 27$.
[2] Shigemura K, ArakawaS, Miura T, Nakano Y, Tanaka K, Fujisawa M. Significance of fluoroquinolone-resistant Escherichia coli in urinary tract infections. Jpn J Infect Dis 2008; 61: 226-8.

[3] van der Mee-Marquet N, Savoyen P, Domelier-Valentin AS, Mourens C, Quentin R. CTX-M-type fluoroquinolone-resistant Escherichia coli: analysis of the colonization of residents and inanimate surfaces 1 year after a first case of urinary tract infection a nursing home in France. Infect Control Hosp Epidemiol 2010;31: 968-70.

[4] van der Starre WE, van Nieuwkoop C, Paltansing S, et al. Risk factors for fluoroquinolone-resistant Escherichia coli in adults with community-onset febrile urinary tract infection. J Antimicrob Chemother 2011;66(3): 650-6.

[5] Rattanaumpawan P, Tolomeo P, Bilker WB, Fishman NO, Lautenbach E. Risk factors for fluoroquinolone resistance in Gramnegative bacilli causing healthcare-acquired urinary tract infections. J Hosp Infect $2010 ; 76: 324-7$.

[6] Urban C, Mariano N, Bradford P A, et al. Identification of CTX-M ß-lactamases in Escherichia coli from hospitalized patients and residents of long-term care facilities. Diagn Microbiol Infect Dis 2010; 66: 402-6.

[7] Lewis JS, Herrera M, Wickes B, Patterson JE, Jorgensen JH. First report of emergence of CTX-M-type extended-spectrum Blactamases (ESBLs) as the predominant ESBL isolated in a U.S. 
health care system. Antimicrob Agents Chemother 2007; 51: 401521.

[8] Castanheira M, Mendes RE, Rhomberg PR, Jones RN. Rapid emergence of $b l a_{\mathrm{CTX}-\mathrm{M}}$ among Enterobacteriaceae in U.S. medical centers: molecular evaluation from the MYSTIC Program (2007). Microb Drug Res 2008; 14: 211-6.

[9] Pitout JD, Laupland KB. Extended-spectrum B-lactamaseproducing Enterobacteriaceae: an emerging public-health concern. Lancet Infect Dis 2008; 8: 159-66.

[10] Clinical and Laboratory Standards Institute (CLSI), Performance standards for antimicrobial susceptibility testing; Twenty-first informational supplement, document M100-S21. Clinical Laboratory Standards Institute, Wayne, PA 2011.

[11] Zhanel GG, Hisanaga T L, Laing N M, et al. Antibiotic resistance in Escherichia coli outpatient urinary isolates: final results from the
North American Urinary Tract Infection Collaborative Alliance (NAUTICA). Int J Antimicrob Agents 2006; 27, 468-75.

[12] Lagace-Wiens PR, Nichol KA, Nicolle LE, et al. ESBL genotypes in fluoroquinolone-resistant and fluoroquinolone-susceptible ESBL-producing Escherichia coli urinary isolates in Manitoba. Can J Infect Dis Med Microbiol 2007; 18: 133-7.

[13] Mac Aogáin M, Mooij MJ, Adams C, Clair J, O'Gara F. Emergence of extended-spectrum beta-lactamase and fluoroquinolone resistance genes among Irish multidrug-resistant isolates. Diagn Microbiol Infect Dis 2010; 67: 106-9.

[14] Karlowsky J A, Hoban DJ, DeCorby MR, Laing NM, Zhanel GG. Fluoroquinolone-resistant urinary isolates of Escherichia coli from outpatients are frequently multidrug resistant: Results from the North American Urinary Tract Infection Collaboratory AllianceQuinolone Resistance study. Antimicrob Agents Chemother 2006; 50: 2251-4.

(C) Bouchillon et al.; Licensee Bentham Open.

This is an open access article licensed under the terms of the Creative Commons Attribution Non-Commercial License (http://creativecommons.org/licenses/ by-nc/3.0/) which permits unrestricted, non-commercial use, distribution and reproduction in any medium, provided the work is properly cited. 